\section{Multiple Learning Mechanisms in the Development of Action}

\author{
KAREN E. ADOLPH, NEW YORK UNIVERSITY, \\ AND AMY S. JOH, DUKE UNIVERSITY
}

a broad range of new problems. A second mechanism, association learning, links particular cues with consequences. Unlike learning sets, association learning is not optimal for dealing with novelty and variability because transfer of learning is limited to a narrow range of problems that shares similar cues and contexts. Given its limitations, association learning may serve as a fallback mechanism when learning sets are not viable.

Our argument is not that learning sets and association learning are the only mechanisms for controlling balance and locomotion - other learning mechanisms may also play a role. Rather, we suggest that relying on these two learning mechanisms is necessitated by the nature of the available perceptual information for controlling motor actions. We garner evidence for learning from the four postural milestones in infant development: sitting, crawling, cruising, and walking. We focus on balance and locomotion because postural control is the foundation for all motor actions involving the torso and extremities (Reed, 1982, 1989; Bertenthal \& Clifton, 1998; Adolph \& Berger, 2005, 2006).

In the first part of the chapter, we set the stage for our argument by describing different sources of perceptual information that specify variations in surface layout (the shape and the dimensions of the path) versus variations in friction and rigidity (the resistance of the ground surface against the body). Different types of exploration are required to generate the necessary visual and tactile information for surface layout and for friction and rigidity. In the second section, we describe the signature characteristics of learning sets and association learning. We argue that learning sets are an optimal mechanism for responding adaptively to variations in surface layout but not to variations in friction and rigidity and that the converse is true for association learning. Our account leads to different predictions regarding the time course of learning and the breadth of transfer of learning to new problems for surface layout and for friction and rigidity. In the subsequent sections, we outline the evidence for the separate learning mechanisms: learning sets for surface layout and association learning for friction and rigidity. Finally, we conclude the chapter by revisiting the two learning mechanisms and placing them in relation to broader issues of learning and development.

\section{EXPLORATION OF SURFACE LAYOUT VERSUS FRICTION AND RIGIDITY}

A central issue for postural control is the problem of selecting the appropriate actions for moving through a variable environment. Two types of environmental changes are particularly relevant for controlling balance and locomotion. One type of change, variations in surface layout, affects possibilities for stance and locomotion by changing the shape of the path in three dimensions: the narrow and winding path between the piles of laundry littering the floor, the steep stairs leading down to the garage, the low gate on which visitors bang their heads, and the sloping road before the intersection. A second type of change, variations in the material properties of each ground surface-footwear pairing, affects friction and rigidity by changing the size of the necessary. resistive forces for friction and 
rigidity conditions: the smooth bathroom tiles and the wet feet that slide over them, the deformable piles of laundry and the surprisingly firm mattress on the bed, and the slippery stairs leading down to the dusty street.

\section{Novelty and Variability in Postural Development}

For infants, the problem of coping with variability and novelty is heightened because learning is nested in the context of developmental change. Infants' bodies and skills change dramatically over the first two years of life (for review, see Adolph \& Berger, 2005). They experience rapid fluctuations in muscle mass and fat. Their overall body dimensions become leaner and less top-heavy. Their height and weight increase in large and sudden spurts; for example, infants can grow 0.5 to 1.5 centimeters in a single day (Lampl, Veldhuis, \& Johnson, 1992). Their proficiency at balance and locomotion shows rapid improvements and decrements as they discover new strategies for moving their bodies from place to place and for transitioning between postures. Typically, improvements are most striking in the first few months after the onset of a new locomotor skill (for review, see Adolph \& Berger, 2006). All of these developmental changes alter the biomechanical constraints on movement, creating novel and variable situations for maintaining balance and locomoting through the environment.

Moreover, infants' bodies and skills change in a unique way during the first years of life. Infants acquire new postural-control systems in the course of development (Adolph, 2002, 2005). Figure 7-1 illustrates four major postural-control systems in infant development: sitting (unsupported with the legs outstretched), crawling (on belly and on hands and knees or feet), cruising (moving sideways in an upright posture, holding onto furniture or a railing for support), and walking (with the hands free, while facing forward). These four postures typically appear between 6 and 16 months of age, in the illustrated order. However, the sequence can vary among infants and cultures (e.g., some infants skip crawling altogether), and the age range at skill onset is extremely wide (Frankenburg \& Dodds, 1967; Hopkins \& Westra, 1988, 1989, 1990). The important point with

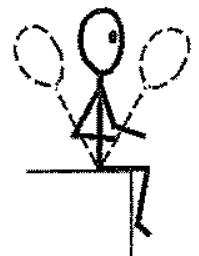

Sitting

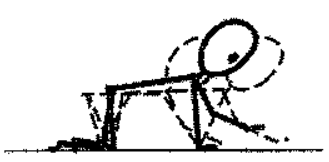

Crawling

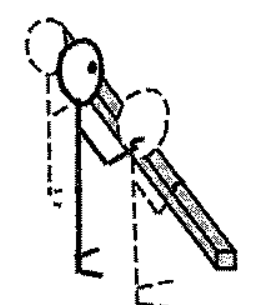

Cruising

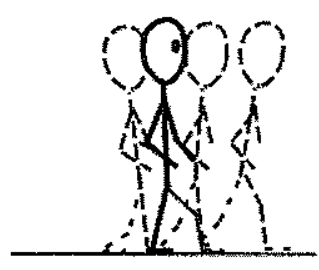

Walking
Figure 7-1. The four postural control systems in their typical order of development. Each posture denotes a distinct problem space that requires unique strategies for obtaining relevant perceptual information, keeping balance, and locomoting through the environment. regard to novelty is that no infant acquires all four postures simultaneously. The postures appear staggered over several months so that at each point in development, infants are experts in an earlier developing posture and novices in a later developing one.

Each postural-control system represents a different problem space defined by a unique set of parameters for maintaining balance. Each posture has a different key pivot around which the body rotates (the hips for sitting, the wrists for crawling, the shoulders for cruising, and the ankles for walking) and a different region of permissible postural sway within which the body can rotate before falling (Fig. 7-1). There are different muscle groups for keeping the body upright and for propelling it forward, different vantage points for viewing the ground ahead, different correlations between visual and vestibular information, different access to mechanical information from touching the ground, and so on (Adolph, 2002,2005 ). In short, each posture requires moving different body parts and is controlled by different sources of perceptual information.

Developmental changes in infants' bodies and skills bring about corresponding changes in the environment. New postures and vantage points and new and improved forms of mobility open up a new world of opportunities for learning. As infants gain access to new places and surfaces, they experience aspects of the environment for the first time. Stairs, slopes, furniture, corners, and doorways present new variations in surface layout. Carpet, wood, tile, grass, sand, ice, and water present new friction and rigidity conditions. As E. J. Gibson (1992) put it, watching a child discover a playground for the first time is a revelation in attention to new affordances for action. For a crawling infant, the playground slide may be a shelter to sneak beneath. For a cruiser, the same apparatus provides poles, stairs, and edges to support pulling to a stand and practicing upright skills. For a toddler, the sliding surface offers opportunities for climbing upward and multiple means for descent.

\section{Exploration}

In the face of so much variability and novelty, how might infants keep balance during stance and locomotion? Perceptual exploration is the key to adapting to changing constraints on action (J. J. Gibson, 1979; E. J. Gibson, 1988; E. J. Gibson \& Pick, 2000; von Hofsten, 2003, 2004). Exploration provides feedback about the consequences of the last movement and informs infants' decisions about what to do next. Movements of the eyes, head, torso, arms, and legs generate perceptual information about the status of the body, the features of the environment, and the relationship between the two. Optic flow, for example, generated by whole-body movement during locomotion specifies the speed and direction of movement and the time to contact a distant obstacle (Lee, 1993). Turning the head to peer over the edge of a precipice or around a corner generates motion parallax providing depth cues for perceiving the surface layout. Rubbing the foot along the ground provides tactile information about friction and rigidity. Note that exploration is not sufficient for adaptive responding-infants might see or feel an obstacle and plunge forward nonetheless-but exploratory movements 
are necessary for generating the requisite perception information to guide appropriate motor decisions.

Exploratory movements can be deliberate, such as when infants poke out their feet to test an obstacle in the path. However, more frequently, exploration is incidental, such as when crawlers' hands touch a new surface in the course of crawling or when walking movements generate information for friction underfoot (Adolph, Joh, \& Eppler, 2008; Joh, Adolph, Narayanan, \& Dietz, 2007). Because the eyes are "parked" in front of the face, incidental visual exploration typically provides the first source of information about an upcoming obstacle (Patla, 1998). If a happenstance glance indicates that something may be amiss, focused and deliberate exploratory movements will follow (Adolph, Eppler, Marin, Weise, \& Clearfield, 2000). The velocity and amplitude of forward movements decrease, body sway increases, head and eyes point directly at the potential obstacle, and various means may be employed for engaging in tactile explotation with the hands or feet (e.g., positioning the body to probe the surface with a foot, grabbing a handhold to augment balance during the probe).

Locomotor movements involve large displacements of the body. Walkers require a relatively long response period and multiple walking steps to modify gait appropriately in preparation for an upcoming obstacle, whether they dampen forces to brace themselves for contact with the obstacle, increase forces to leap over the obstacle, or redirect forces to veer around it (Patla, 1989; Patla, Robinson, Samways, \& Armstrong, 1989; Patla, Prentice, Robinson, \& Neufeld, 1991). Thus, prospective trol of locomotion depends on reliable visual cues from a distance to instigate the sequence of more focused exploration (Adolph et al., 2000; Adolph et al., 2006) There is no teason to modify current gait patterns, stop to engage in tactile exploration, or search for alternative routes or methods of locomotion if incidental visual exploration does not alert walkers to a potential threat to balance. Without advance warning, prospective control is impossible. Walkers might plant an unsuspecting foot on the obstacle and the only recourse is raspecting foot on the obstacle, and the only recourse is reactive adjustments (e.g., waving the arms and quickly bending the torso to try to avoid or mitigate a fall).

\section{Information for Surface Layout Versus Friction and Rigidity}

The sequential process of exploration is well suited for coping with variations in surface layout, but not for variations in friction and rigidity. The difference stems from the nature of the perceptual information. Changes in the shape and dimensions of the path are specified by reliable visual cues, whereas changes in friction and rigidity are not (Adolph et al., 2000; Joh, Adolph, Campbell, \& Eppler, 2006). Friction and rigidity are resistive forces that emerge only when two surfaces come into contact, such as when the foot presses against the ground during walking. In addition, the manner of contact between the surfaces (e.g., the velocity and angle of the foot as it touches the ground) determines the size of the resistive forces. Because friction and rigidity result from the size of the resistive forces. Because friction and rigidity result from the interaction between two surfaces, single surface cannot serve as a cue for friction and rigidity conditions. The same shiny wooden floor may be slippery or resistive, depending on the walker's footwear and walking patterns.
Moreover, visual cues for friction and rigidity are variable and context-dependent. Slippery surfaces can be shiny or matte, dark or light. Deformable surfaces can be bumpy or smooth, patterned or plain. Even shine, which adult walkers use most frequently as a visual cue for slip, is not reliable for specifying friction conditions because there is no such phenomenon as "shine constancy." Shine perception is strongly affected by the color of the ground surface, overhead lighting conditions, and viewing distance and angle--factors that do not affect the actual friction condition (Joh, Adolph, Campbell et al., 2006). Adults rate dark surfaces as looking shinier and more slippery compared with white surfaces, even when gloss levels are equal across displays. They rarely judge white surfaces as looking shiny or slippery unless they view the surface from directly above - too late to modify walking patterns in preparation for stepping onto the surface. In other words, for detecting novel variations in friction and rigidity, there are no reliable visual cues.

\section{TWO LEARNING MECHANISMS IN \\ THE DEVELOPMENT OF ACTION}

Differential availability of visual information from a distance leads to a reliance on two different learning mechanisms-learning sets for surface layout and association learning for friction and rigidity. In a perfect world, where all obstacles are reliably specified by visual cues from a distance, presumably, infants would rely solely on learning sets - the mechanism that promotes the broadest transfer to novel challenges for postural control. However, in our imperfect world, where novel variations in friction and rigidity cannot be specified reliably with visual cues, infants must also rely on association learning. The price of relying on a learning mechanism with narrow transfer is increased errors in prospective control in novel situations.

\section{Learning Sets}

An optimal learning mechanism for coping with variability and novelty is the learning set. Our notion of learning sets is based on Harlow's (1949, 1959; Harlow \& Kuenne, 1949) idea that acquiring the ability to solve problems is more effective for a broader range of situations than learning particular solutions for particular problems. Like the adage, "Give a man a fish and you feed him for a day; teach a man to fish and you feed him for a lifetime," learning sets provide a means rather than a quick fix. With learning sets, learners acquire a set of informationgenerating behaviors and heuristic strategies for figuring out solutions to novel problems rather than for learning simple cue-consequence associations or facts (Stevenson, 1972). As Harlow put it, they are "learning to learn." With learning sets, transfer is limited only by the boundaries of the particular problem space. Accordingly, acquiring a learning set entails recognizing the problem space, identifying the relevant parameters for operating within it, acquiring the exploratory procedures and strategies for generating the appropriate information, and knowing how to use the resulting information to solve the problem at hand. 
For example, in Harlow's (1949; Harlow \& Kuenne, 1949; Harlow, 1959) original studies, adult monkeys acquired learning sets to solve discrimination problems. Monkeys were required to choose a target object from a pair of objects that differed on multiple characteristics (e.g., small green triangle and large yellow cylinder) and shifted in their left-right positions. The target object yielded a food reward. After a block of 10 trials, the experimenter introduced a new pair of objects (e.g., striped rectangle and polka-dot star). At first, the monkeys searched haphazardly or learned associations between cues and the food reward (e.g., polka-dot stars hide raisins). The process continued over hundreds of trial blocks until monkeys could find the food reward with new pairs of objects after only one presentation of a new pair of objects. Instead of using trial and error or cue-consequence associations, they had acquired a learning set: They learned to explore one object by lifting it and to track the one in the pair that covered the reward, a sort of win-stay/lose-shift rule, for the remaining trials in the block. Altogether, learning to learn for discrimination problems meant that the monkeys recognized the pairs of objects as the problem space, identified the perceptual qualities of the objects as the relevant parameters (color or shape rather than their spatial position), possessed the necessary exploratory procedures to visually differentiate the two objects and search the wells beneath them, abstracted the win-stay/lose-shift strategy, and used the information to track the food reward.

Learning sets have three important characteristics. First, within the particular problem space, learning sets allow a broad transfer of learning to novel problems. For example, after acquiring a learning set for discrimination problems, Harlow's monkeys demonstrated perfect performance with pairs of completely novel shapes. Thus, learning sets are a far cry from mere stimulus generalization. With learning sets, monkeys had acquired a means for coping with novelty. Note, however, that in Harlow's simple model system, the problem space was extremely small: Monkeys with expertise solving discrimination problems showed no ability to solve oddity problems and vice versa. The learning set was limited to the type of problem that defined that problem space.

In the immensely more complex task of infants' balance and locomotion, each postural-control system operates like a distinct problem space. Within a posture, the range of problems is enormous. Every movement on every surface constitutes a different problem for postural control. Every change in infants' body growth and skill level creates different biomechanical constraints on balance and locomotion. For each postural-control system in development, learning to learn would mean that infants must recognize the new posture as a new problem space, identify the relevant parameters for keeping balance, acquire the appropriate exploratory procedures to determine the current constraints on balance, and learn to use the resulting information to calibrate the settings of the parameters on line (Adolph, 2002; Adolph \& Eppler, 2002; Adolph, 2005). After acquiring a learning set for walking, for example, infants should be able to maintain balance during stance and locomotion when faced with variations in the layout of the terrain, while carrying loads and wearing different clothes and shoes, after developmental changes in body growth and skill levels, and in different goal contexts. The scope of transfer should be limited only to the boundaries of the upright postural-control system.
Just as learning sets do not transfer between discrimination problems and other types of problems (e.g., oddity), learning sets should not transfer between postural-control systems. Infants must acquire separate learning sets for sitting, crawling, cruising, and walking. Because the parameters, exploratory movements, and relations between perceptual information and the various parameters differ among postural systems, learning sets for earlier developing postures should not transfer to later developing ones. The ability to respond adaptively to variations in surface layout while crawling, for example, should not help infants after they begin walking.

The second distinguishing characteristic of learning sets is that acquisition is extremely slow. In general, the larger the problem space, the slower the acquisition. Harlow's adult monkeys required dozens of blocks of trials with different pairs of objects-several thousand trials - distributed over multiple sessions to acquire learning sets for discrimination problems (Harlow, 1949; Harlow \& Kuenne, 1949; Harlow, 1959). And this was with a relatively simple task. Exploratory procedures for searching under the objects were simple, few, and already existed in monkey's repertoires. Monkeys could solve the discrimination problems by applying a fixed exploratory strategy and a win-stay/lose-shift rule.

Learning to learn in discrimination problems is easy compared to acquiring learning sets for postural-control systems, which requires enormous amounts of experience. Because balance and locomotion occupy an immense problem space for each posture, human infants might require massive amounts of experiencehundreds of thousands of "trials" of balance shifts and steps-over weeks or months to acquire a learning set to cope with the exigencies of postural control in everyday life. Indeed, recent research suggests that infants' everyday locomotor experience is a mini marathon. The quantity of experience is sufficiently massive to facilitate the acquisition of learning sets (Adolph \& Berger, 2006). Fourteen-month-old toddlers, for example, typically take more than 2,000 steps in the course of an hour; strung together, end to end, infants walk the length of more than 40 football fields in the course of a day (Garciaguirre \& Adolph, 2006) in a variety of contexts, differing in location, goal, and social interaction.

Compared to the discrimination problems in Harlow's model system, the problem space for postural control is extremely broad. Infants must work to keep their bodies in balance during all of their waking hours. Crawlers are engaged in balance and locomotion for approximately 5 hours per day, and walkers for approximately 6 hours (Adolph, 2002). Infants travel through all of the open rooms in their homes, averaging exposure to 12 different ground surfaces per day (Chan, Biancaniello, Adolph, \& Marin, 2000). Exploration in the service of balance and locomotion is complex and varied. Many-perhaps an infinite number-of exploratory movements of eyes, head, and body yield information in visual, tactile, and proprioceptive modalities.

Moreover, for each postural-control system, infants must discover and hone the exploratory swaying, looking, and touching movements that are required to generate perceptual information for balance and locomotion. Rather than learning fixed strategies or rules, learning to gauge the current constraints on balance requires infants to detect dynamic and probabilistic functions. In fact, everyday 
experience with balance and locomotion may impede learning about fixed rules and thereby facilitate the acquisition of learning sets. On average, 14-month-old walking infants lose their balance and fall 15 times per hour, which suggests that they may fall more than 90 times per day (Garciaguirre \& Adolph, 2006). Falls are not related to the appearance of the ground surface because they are not precipitated by variations in surface layout or in friction and rigidity. Most falls are simply a byproduct of the variability in infants' own motor systems.

The third critical characteristic of learning sets is a reliance on adequate perceptual information to elicit the appropriate exploratory procedures and strategies in the learning set. In Harlow's discrimination task, monkeys relied on visual information about object properties to guide their manual search. Similarly, learning sets for postural-control systems require visual information from a distance to prompt more focused exploratory movements to gauge upcoming threats to balance. Learning sets are an optimal solution for coping with novel changes in surface layout because infants can see changes in surface layout from a distance. Visual depth cues (e.g., motion parallax, convergence, texture gradients) can reliably specify stairs, slopes, narrowing of the path, and so on. These visual cues are sufficient to prompt infants to modify their ongoing locomotion, slow down, direct their attention to the obstacle, and, if necessary, begin searching for an alternative solution.

Unlike variations in surface layout, novel changes in friction and rigidity are not specified by reliable visual cues. Thus, infants who have laboriously acquired learning sets for postural control-and adults who have had learning sets for upright posture for dozens of years - are not likely to execute the full range of exploratory movements in their repertoires based only on visual information about friction and rigidity. Accordingly, variations in friction are a leading cause of accidental injury from falling in people across all ages and stages of learning and development (National Safety Council, 2003). Rather than controlling balance prospectively, people are most likely to be surprised by novel changes in friction and rigidity and forced to respond reactively after stepping haphazardly onto the offending surface.

\section{Association Learning}

Association learning is a fallback mechanism for coping with variations in friction and rigidity because fixed associations are not well suited for controlling balance and locomotion under novel and variable conditions. By association learning, we mean pairings between cues and consequences. When two events co-occur, they can become associated (e.g., stepping onto a white, shiny patch of ground on a cold, winter day results in slipping); with repeated pairings, the association between the cue and the consequence becomes stronger. In principle, associated pairings could lead to a stereotyped motor response (e.g., see ice patch, walk around it) or to a variety of appropriate responses (leap over it, step carefully onto it, coast over it, etc.). Moreover, the exploratory behaviors in a learning set could mediate between the learned association and the motor decision, and vice versa. That is, associations between particular cues and consequences can instigate the exploratory procedures and strategies in a learning set by shifting attention to the relevant cues in similar situations (e.g., after slipping on an ice patch, becoming vigilant about potential ice patches during the remainder of the walk). Conversely, initiating the exploratory procedures in a learning set can lead to forming a learned association (e.g., tactile exploration of a steep stair can reveal the potential for slipping and lead to the formation of an association between the sight of the stair and the aversive consequence).

Given their reliance on particular cues or a class of cues, association learning and learning sets share an important similarity: Prior experiences with irrelevant cues can impede learning when the cues subsequently become relevant. For example, after initially experiencing a particular cue - a clicker sound-without any consequences, rats exhibit learned irrelevance. Later, they take longer to associate the clicker sound with a shock. They require more pairings of the clicker and shock to display a freezing response compared with rats that had not previously experienced the uncorrelated click (Baker \& Mackintosh, 1979). With learning sets, animals learn to ignore a class of cues. Harlow's monkeys learned to ignore the spatial location of the target shapes while acquiring learning sets for discrimination problems. If this previously irrelevant type of cue were to become relevant, monkeys would find it more difficult to incorporate it into the learning set.

In the more complex case of balance and locomotion, infants may learn to ignore cues such as the color, texture, and shine of the ground surface because this class of cues (i.e., visual appearance of the ground surface) is not typically relevant for balance and locomotion. Although variations in the ground may cause infants to fall, infants' falls are not usually caused by variations in the ground surface; most falls occur on a flat, rigid, high-traction surface (Garciaguirre \& Adolph, 2006). Moreover, information that was previously irrelevant for one postural-control system (e.g., tactile, underfoot information for crawling) may impede acquisition of a learning set for a later developing posture in which the information becomes relevant (e.g., cruising and walking). Learned irrelevance changes motivation and attention. After learning that a cue is not correlated with the consequence, animals stop looking for the correlation and stop attending to the cue.

Association learning differs from learning sets in three important ways. First, association learning requires an initial encounter with the particular cue-consequence pairing. In novel situations, it is impossible to respond adaptively on the first trial. Walkers must experience wet feet from stepping into a puddle and a slip from stepping onto a patch of ice in order to avoid these consequences in the future. In contrast, learning sets can function in novel situations without a history of prior errors. Monkeys solved new discrimination problems on the first trial (Harlow, 1949; Harlow \& Kuenne, 1949; Harlow, 1959), and experienced infants can determine whether a new configuration of the surface layout is safe or risky on their first encounter (e.g., Adolph, 1997).

Second, with association learning, transfer of learning is relatively narrow. Transfer is limited to the particular cues in the learned pairing and the context in which the pairing was formed. Rats, for example, freeze with fear in a corner of their cage after learning to associate a tone with a shock. But, they ignore 
a different tone that results in the same consequence, and they ignore the same tone when placed into a different cage. However, when exposed to the original tone in the original cage, the rats freeze once again (Bouton \& Bolles, 1979). Likewise, in the case of balance and locomotion, cue-consequence associations can guide adaptive motor decisions only when new cues and contexts are sufficiently similar to the learned ones (e.g., on a cloudy, cold day, the patch of ice may not appear shiny; on a sunny, warmer day, walkers may ignore shiny regions of sidewalk) and when the current status of the body does not change the consequences (ice is not slippery if walkers are wearing crampons).

A third important difference between association learning and learning sets is that associated pairings are relatively easy to form. Infants and many species of animals can learn multiple, complex, hierarchical associations that elicit adaptive responses in the presence of the appropriate cues and contexts. Moreover, under optimal training conditions, association learning can be fast, requiring only a fraction of the trials needed for learning sets. For example, in a classic task (Lashley, 1930), rats stood on a raised jumping stand facing two cues varying in size, shape, color, or pattern. One cue marked a door that opened when the rats jumped headfirst into it; the other marked a solid wall into which the animals crashed, falling into a safety net below. Given the salience of the consequence, rats learned to identify the relevant cue at $100 \%$ correct performance after only 20 to 30 trials. Under optimal conditions, with a single, high-contrast stimulus to mark the door (e.g., a white square versus a black one), rats showed $100 \%$ correct performance after only 4 trials.

Similarly, under the appropriate circumstances, human infants show fast association learning. Three-month-olds learn to associate their foot kicks with the jiggling of an overhead mobile after only a few minutes of practice (Rovee \& Rovee, 1969; Lewis, Alessandri, \& Sullivan, 1990). In the typical set-up, infants lie supine with a ribbon tied from one ankle to the mobile. Spontaneous leg movements cause the colorful mobile elements to move and to jingle and clack together. Distinctly colored and patterned bumpers lining the walls of the crib optimize the speed of learning by making the context more salient and distinct.

\section{Summary of Dissociations Between Learning Sets and Association Learning}

Experience-related improvements in infants' motor decisions are crucial to both learning sets and association learning, and both mechanisms rely on the appropriate cues to instigate an adaptive response. However, the two learning mechanisms lead to different predictions about patterns of learning for surface layout and for
friction and rigidity.

For surface layout, a learning-set mechanism would predict a long, slow acquisition period before infants could respond adaptively. Acquiring a learning set should require weeks or months of practice for each postural-control system in development. Infants should be indifferent to feedback from errors in a single test session. Improvements in the accuracy of infants' motor decisions should be gradual, as new exploratory behaviors and strategies enter infants' repertoires and become more finely honed. However, once a learning set is acquired, experienced infants should make adaptive motor decisions on their first encounter with a novel obstacle (e.g., a steep slope or narrow bridge) in their experienced posture. Visual depth cues from a distance should elicit an array of focused, exploratory behaviors. Infants should be able to update their assessment of their own abilities in accordance with changes in functional body dimensions and level of locomotor skill. For example, they should correctly treat the same slope or bridge differently depending on the current biomechanical constraints. However, learning sets predict no transfer of learning between postures. Infants should display more adaptive responding in their more experienced posture, even when tested in both postures on the identical slope or narrow bridge.

An association-learning mechanism would make very different predictions about coping with variability and novelty in the surface layout. Learning should be relatively fast, possibly occurring in a single test session under optimal conditions with salient cues and consequences. Even for the most experienced infants, learning should require errors, at least on the first encounter with a novel obstacle, and errors should decrease with repeated encounters. Improvements in the accuracy of infants' motor decisions should be all or none, depending on whether infants have formed the appropriate association. However, learning should be restricted to the particular cues and context of the training situation. Learning may not transfer, for example, from shallow to steep slopes, or from slopes to drop-offs and bridges, even within the same posture. When cues or contexts change, infants should again show a lack of adaptive responding. Given similar visual cues about the status of the surface layout, association learning would predict that infants treat the same slope or bridge similarly, regardless of the current biomechanical constraints on action. They should not update their motor decisions in accordance with changes in their bodies and skill levels; they should show no decrement over developmental transition in postural milestones. Thus, in an experienced posture, infants' responses to changes in body dimensions and skill levels should be less accurate if they are relying on learned associations than on learning sets. But over the transition from experienced postures to later developing ones, responses should be more accurate.

An association-learning mechanism makes identical predictions for how infants should cope with variations in friction and rigidity as for variations in surface layout: Infants should show fast, all-or-none learning of particular cues and consequences following an initial encounter. Predictions of learning sets, however, differ between these two types of environmental changes. According to a learningset account, infants should frequently fail to respond adaptively to novel changes in material substance because friction and rigidity are not specified reliably by visual cues from a distance. Without a visual prompt, infants are not likely to use all of the exploratory procedures and compensatory strategies in their learning set. In addition, learning sets and association learning share a common prediction for friction and rigidity: Infants may err in multiple encounters with an obstacle because they have learned to ignore visual cues such as color, texture, and shine in the formation of a learning set. The fact that falling is typically unrelated to the appearance of the ground surface may lead to learned irrelevance. 


\section{EVIDENCE FOR LEARNING SETS: COPING WITH CHANGES IN SURFACE LAYOUT}

In dozens of studies, researchers have observed how infants respond to changes in the surface layout that pose a challenge for balance and locomotion (reviewed in Bertenthal \& Clifton, 1998; Adolph \& Berger, 2005; Adolph \& Berger, 2006). Here, we describe a subset of these studies that were designed to test the predictions of learning sets (reviewed in Adolph, 2002; Adolph \& Eppler, 2002; Adolph, 2005). The studies shared a common logic and procedure: Infants were observed as they encountered novel challenges to balance and locomotion at the edge of an adjustable slope or gap in the surface of support (Fig. 7-2, panels A-E). The slant of the slope $\left(0^{\circ}\right.$ to $\left.90^{\circ}\right)$ or width of the gap $(0 \mathrm{~cm}$ to $90 \mathrm{~cm})$ varied in continuous gradations from trial to trial. A motorized mechanism varied the degree of slope by moving a landing platform up and down; a platform varied gap width by sliding back and forth along a calibrated track. Caregivers stood at the bottom of the incline or the far side of the gap and encouraged their infants to traverse the obstacle. A highly trained experimenter followed alongside infants to ensure their safety if they began to fall.

In the first part of each test session, we used a psychophysical staircase procedure to determine the limit of each infant's ability - a motor threshold (e.g., Adolph, 1995, 1997, 2000). Increments of greater difficulty were presented after successful trials (infants safely traversed the obstacle) and easier increments were presented after unsuccessful trials (infants fell or refused to cross) in an up-anddown pattern (hence, the "staircase") until we identified the steepest slope or widest gap that infants could navigate successfully on the majority of trials. The estimates of individualized motor thresholds allowed us to assess the adaptiveness of infants' motor decisions relative to their current physical abilities. This normalization process was crucial because at the same age, infants show large individual differences in abilities, and their abilities change dramatically from week to week. A slope or gap that is safe for a more skilled infant might be risky for a poorly skilled one; what might be a risky slope one week might be easily navigated the following week when infants' abilities improve.

In the second part of the session, the experimenter presented probe trials at safe and risky increments (i.e., within and beyond infants' ability, respectively). Adaptive decisions for action require infants to detect the current limits of their own bodies and skills relative to incremental changes in the degree of slant or the width of the gap. That is, infants should attempt safe increments within their ability and select alternative strategies or avoid risky increments beyond their ability. Thus, the role of everyday experience with each posture in adaptive motor decisions was of central interest for evaluating the learning-set account. To determine the informational basis of infants' motor decisions, we examined their exploratory activity before selecting a motor response- their latency to initiate traversal and their exploratory looking and touching behaviors. To assess behavioral flexibility in infants' responses, we observed the type and number of strategies infants used for coping with risky obstacles-whether they simply avoided the obstacle or generated alternative methods of traversal.
A.

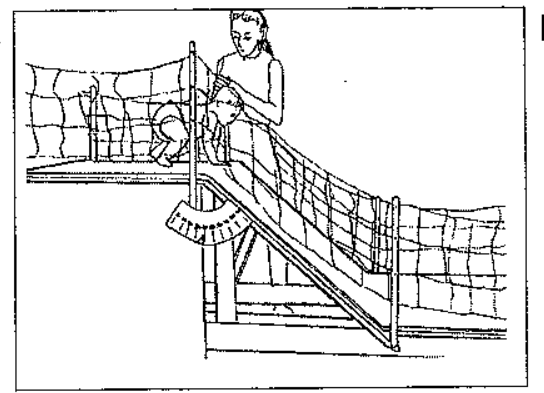

B.

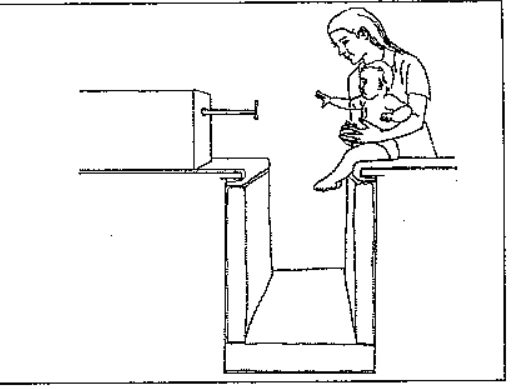

C.

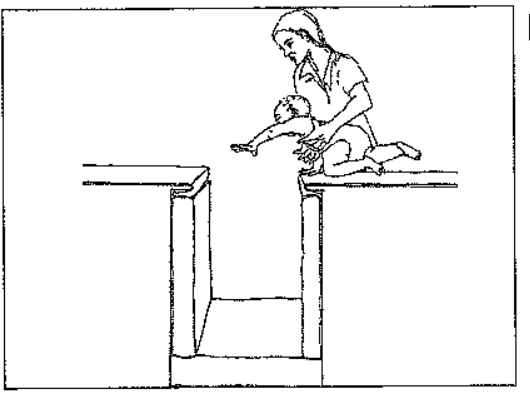

D.

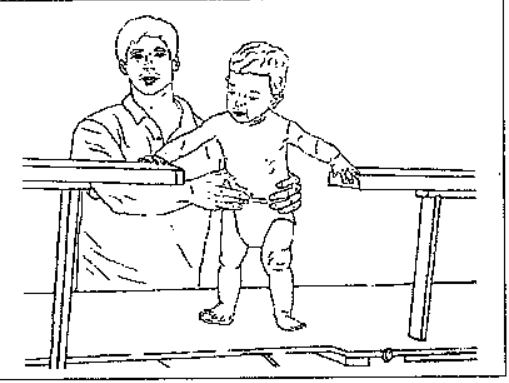

E.
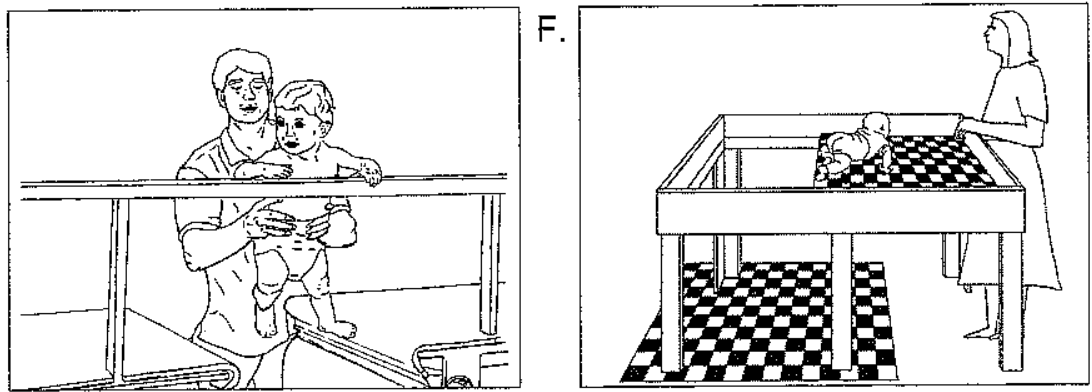

Figure 7-2. Tasks and apparatuses used in studies of learning sets. (A) Crawling and walking infants descending a slope. (B) Sitting infants leaning forward to reach for toys at the edge of a gap in the floor. (C) Crawling infants leaning forward to cross over a gap in the floor. (D) Cruising infants spanning a gap in the handrail. (E) Cruising infants crossing over a gap in the floor. (F) Crawling and walking infants on the visual cliff. All apparatuses, except for the visual cliff, were adjustable in continuous increments.

\section{Slopes}

One way to test the learning-set account is to observe infants longitudinally, as they transition from one postural-control system to another. Accordingly, infants were observed every three weeks, from their first week of crawling until thirteen weeks after they began walking, on the adjustable sloping apparatus (Fig. 7-2A) (Adolph, 1997). To ensure that improvements in infants' motor decisions were not due to repeated practice with slopes, a control group of infants was tested only 

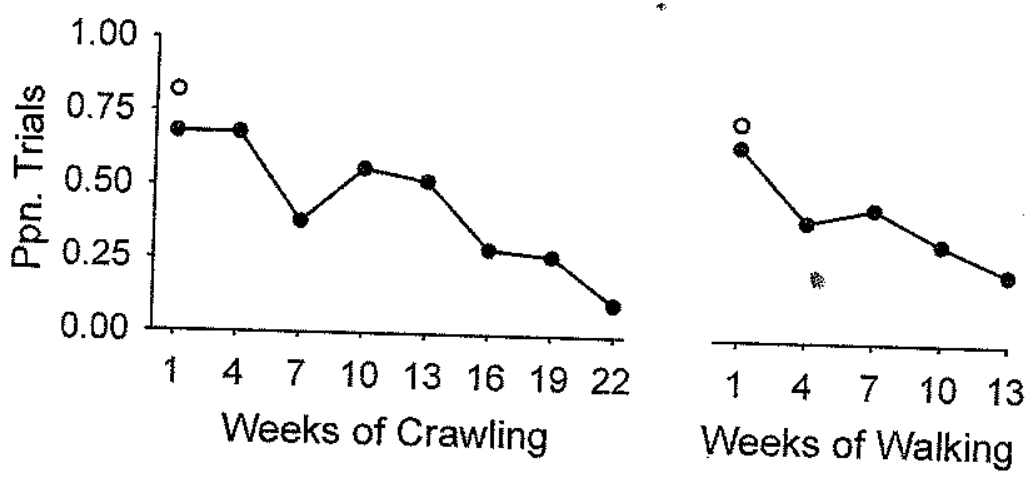

Experimental

Figure 7-3. Proportion of trials on which infants attempted to descend risky slopes and fell. Risky slopes were defined as slopes steeper than infants' individualized motor thresholds. The infants in the experimental group were tested every 3 weeks, from their first week of crawling until 13 weeks after they began walking. tested only 3 times, in their first of walking.

three times: in their first and tenth weeks of crawling and in their first week of walking.

The findings present strong evidence for the acquisition of learning sets for coping with variations in surface layout. As shown in Figure 7-3, in their first weeks of crawling and walking, infants attempted impossibly steep slopes and fell on trial after trial, requiring rescue by the experimenter. The average error rate was $75 \%$ on risky slopes. Over weeks of everyday crawling and walking experience, motor decisions steadily improved. Infants' decisions became more closely aligned to the limits of their own abilities, and their error rates decreased. That is, infants scaled their decisions to the relative amount of risk, an impressive featgiven the dramatic changes in their bodies and skills over the weeks of testing. As in Harlow's (Harlow, 1949; Harlow \& Kuenne, 1949; Harlow, 1959) studies with monkeys, infants learned extremely slowly. Typically, infants required 10 weeks of crawling and walking experience before errors decreased to $50 \%$, and 22 weeks before errors decreased below $10 \%$.

Learning was not the result of repeated practice on slopes. Infants in the control group displayed the same error rates at each of the three matched session times as the infants tested repeatedly, and none of the infants had experience descending playground slides or other slopes outside the laboratory. As further evidence that learning was not dependent on practice descending slopes, crawling infants in several cross-sectional studies showed similar motor decisions and exploratory activity at $8.5,11$, and 12 months of age when age-matched to the infants observed longitudinally; walking infants in cross-sectional research showed similar responses at 12, 14, and 18 months of age (Adolph, Eppler, \& Gibson,
1993; Adolph, 1995; Mondschein, Adolph, \& Tamis-LeMonda, 2000; Adolph \& Berger, 2006; Adolph, Tamis-LeMonda, Ishak, Karasik, \& Lobo, 2008). At the same testing age -12 months--infants showed adaptive motor decisions if they were experienced crawlers but fell down risky slopes if they were novice walkers (Adolph et al., 2008). Learning did not involve a rigid motor-response pattern. Rather, from trial to trial within the same session, individual infants used multiple alternative strategies for coping with risky slopes: sliding down in a sitting position, sliding headfirst prone, backing down feet first, and avoiding descent entirely (Siegler, Adolph, \& Lemaire, 1996; Adolph, 1997).

Moreover, as predicted by learning sets, infants showed no evidence of transfer between crawling and walking. Error rates were just as high in the first week of walking as in the first week of crawling, and learning was no faster the second time around. As shown in Figure 7-3, learning curves for crawling and walking were similar. In fact, learning was so posture-specific that infants showed no evidence of transfer, even from trial to trial. After infants began walking, six consecutive trials on an impossibly steep, 36-degree slope were added to the end of each session. On the first two trials, when infants began in their unfamiliar upright posture, they walked over the brink and fell. On the third and fourth trials, when infants were returned to their familiar crawling posture, they slid down or avoided descent. On the last two trials, when infants started in their unfamiliar walking posture, they again walked over the edge and fell.

Like their motor decisions, infants' exploratory behaviors failed to transfer from crawling to walking. When infants faced slopes from their new upright posture, new parts of their bodies were required to gather information from a new, higher vantage point. In their first weeks of crawling, infants stopped and stared down the slope with their hands over the brink, but plunged down nonetheless. Over weeks of crawling, infants' visual and tactile exploration became increasingly discriminating, fast, and efficient. By their last weeks of crawling, a quick glance and touch on risky slopes was sufficient to support an adaptive motor decision. But when infants began walking, they had to learn new ways of exploring slopes. Experienced walkers either stopped with their feet straddling the brink and rocked back and forth over their ankles or made small stepping movements. After they engaged in visual and tactile exploration, their motor decisions were more accurate. On safe slopes, infants attempted to walk, and on risky slopes they refused.

A second way to test the learning-set account is to probe the extent of transfer within an experienced postural-control system. In this case, we tested 14-monthold walking infants on the adjustable slope. Previous work showed that by 14 months of age, most infants have at least several weeks of walking experience and respond adaptively to variations in surface slant (Adolph et al., 1993; Adolph, 1995). The longitudinal data showed that infants update their assessment of their abilities across naturally occurring changes in their body dimensions and locomotor skill. Here, we experimentally manipulated infants' bodies and skills and observed whether they adapted their motor decisions to their new physical abilities during the testing session (Adolph \& Avolio, 2000). Infants were loaded with lead-weight shoulder packs on some trials and no-weight packs on other trials. 
The lead- and no-weight conditions were interspersed so that infants would have to gauge possibilities for walking at the start of each trial.

As expected, the lead-weight packs made infants' bodies more top-heavy and their balance more precarious. As a result, their walking skill was diminished and their motor thresholds were lower in the lead-weight condition. The critical evidence for learning sets was infants' motor decisions under the two load conditions. Infants correctly attempted to walk down steeper slopes on the no-weight pack trials compared to the lead-weight pack trials. That is, infants updated their motor decisions from trial to trial, based on the current status of the loads in their shoulder packs. They treated the same degree of slope as safe in the no-weight condition and as risky in the lead-weight condition. As in earlier studies, exploratory looking and touching increased with risk level, and infants used a variety of alternative descent strategies to navigate risky slopes.

\section{Gaps}

A third way to test the learning-set account is to compare infants' motor decisions at the same chronological age and in the same novel task in experienced versus novice postures. Accordingly, we tested 9-month-olds in an experienced sitting posture and in a less familiar crawling posture at the edge of an adjustable gap spanning a deep precipice (Adolph, 2000). On average, infants had 3 months of sitting experience but only 1 month of crawling experience. The infants' task was the same in both postures: They were encouraged to lean over the gap to retrieve an attractive toy (Fig. 7-2, panels B-C). Infants began in a sitting or crawling position on a stationary starting platform. A movable landing platform slid back and forth along a calibrated track to create the adjustable gap.

As shown in Figure 7-4, at every risky gap size, infants were more likely to display adaptive responses in their experienced sitting posture than in their less familiar crawling posture. In the experienced sitting posture, infants scaled their motor decisions to the limits of their abilities. They fell into the gap on only $19 \%$ of risky trials, and most errors were clustered at gaps slightly wider than their motor thresholds. In contrast, in the less experienced crawling posture, infants erred repeatedly. They fell into impossibly wide gaps on $61 \%$ of risky trials, and learning was not related to experiences in the laboratory. After falling, infants received the same gap increment on the next trial and fell again $(90 \%$ of repeated trials). When tested in the crawling posture, nearly half of the sample in two experiments plunged headfirst into the widest $(90 \mathrm{~cm})$ gap. Moreover, there was no within-session learning: Infants were equally likely to err in the crawling posture and to respond adaptively in the sitting posture, regardless of whether they were tested in the crawling condition first (where the experimenter rescued them on dozens of trials) or second.

A fourth way to test the learning-set account is to compare infants' motor decisions at the same chronological age in tasks that are relevant to balance and locomotion in an experienced posture versus a later developing posture. In this case, we tested 11-month-old infants who were experienced at "cruising" but who could not yet walk (Leo, Chiu, \& Adolph, 2000). Typically, infants cruise - move
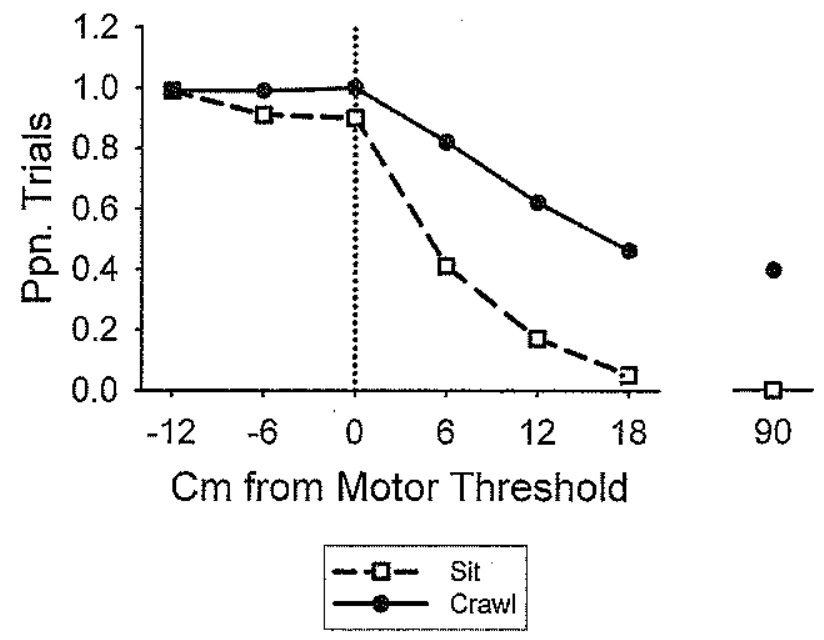

Figure 7-4. Proportion of trials on which infants attempted to cross the gap in the floor and fell. Data are normalized to each infant's motor threshold, represented by 0 on the $\mathrm{X}$-axis. On the $\mathrm{X}$-axis, the negative numbers represent safe gaps (increments smaller than motor thresholds); the positive numbers represent risky slopes (increments larger than motor thresholds).

sideways in an upright posture, hanging onto furniture for support - for a few months before walking. Traditionally, researchers have assumed that cruising and walking are manifestations of the same upright balance control system (McGraw, 1935; Haehl, Vardaxis, \& Ulrich, 2000; Metcalfe \& Clark, 2000). That is, practice cruising teaches infants how to keep balance while walking. However, while cruising, infants use their arms for balance and steering; while walking, infants use their legs. Thus, we put the traditional assumption to the test. We asked whether cruising might be a distinct balance-control system with a separate learning set for coping with potential threats to balance.

In the condition relevant for cruising, infants were encouraged to traverse a continuously solid floor with an adjustable gap in a handrail that they could hold onto for balance (Fig. 7-2D). In the condition relevant for walking, infants were presented with a continuously solid handrail and an adjustable gap in the floor (Fig. 7-2E). In both conditions, an assistant called infants' attention to the obstacle at the start of each trial. In the handrail-gap condition, infants matched their motor decisions to the probability of cruising successfully. For gaps wider than their motor thresholds, infants avoided traversal or crawled to the second handrail. However, in the floor-gap condition, infants appeared to be oblivious to the fact that they need a floor to support their bodies. Most infants attempted to cruise over the widest gaps in the floor, clinging to the handrail as their feet dangled into the precipice. Further evidence for failure to transfer between cruising and walking postures was provided by a group of newly walking infants. New walkers erred in 
both conditions; they attempted to span impossibly wide gaps in the handrail and stepped into impossibly wide gaps in the fioor.

\section{The Visual Cliff}

A recent study with infants on a "visual cliff" posed a challenge to the learningset account (Witherington, Campos, Anderson, Lejeune, \& Seah, 2005). As first devised by E. J. Gibson and Walk (e.g., 1960), the visual cliff is a large glass table, divided in half by a narrow starting board (Fig. 7-2F). On the "shallow" side, a patterned surface is placed directly beneath the glass $(3 \mathrm{~cm})$, providing visual information for a solid surface. On the "deep" side, the patterned surface lies on the floor far below $(90 \mathrm{~cm})$, creating the illusion of an abrupt drop-off. Infants are placed on the starting board and encouraged to cross by caregivers standing at the far end.

To test transfer across developmental changes in posture, 12-month-old crawlers and walkers were tested on the visual cliff in a between-subject design (Witherington et al., 2005). Infants in the crawling group averaged 3.5 months of crawling experience, and all infants in the walking group had less than two weeks of walking experience. In contrast to the prediction of learning sets, the walkers were more likely to avoid crossing the deep side of the visual cliff than the crawlers. However, in another study, infants avoided the apparent drop-off when tested in their experienced crawling posture, but the same infants wheeled straight over the safety glass when tested in an upright posture in a mechanical baby walker (Rader, Bausano, \& Richards, 1980).

Studies using the visual cliff have also yielded conflicting findings regarding the role of locomotor experience (for review, see Adolph \& Berger, 2006). In the most frequently cited study, infants with 6 weeks of crawling experience were more likely to avoid crawling over the deep side of the cliff than infants with only 2 weeks of crawling experience (Bertenthal, Campos, \& Barrett, 1984). The finding is especially striking because infants' age was equated across the two groups. However, other studies found the opposite effect, while controlling for infants' age: Novice crawlers were more likely to avoid the apparent drop-off than experienced crawlers (Richards \& Rader, 1981, 1983).

Albeit famous, the visual cliff is not an optimal paradigm for assessing infants' responses to novel variations in surface layout. Discrepant findings may stem from methodological limitations of the apparatus (Adolph \& Berger, 2006). The safety glass is particularly problematic. For example, because infants have trouble keeping their bodies on the narrow centerboard, some researchers score crossing only after infants have traveled halfway across the safety glass (Sorce, Emde, Campos, \& Klinnert, 1985). As a consequence, findings may be biased toward avoidance responses, although if the safety glass were not there, infants would have tumbled to the floor as soon as their weight shifted off the centerboard. A second problem with the safety glass is that it presents infants with conflicting perceptual information about the deep side of the visual cliff. The drop-off looks risky, but feels safe to the touch. Indeed, the deep side is perfectly safe for locomotion, and infants quickly learn that they can cross it after one or two trials. When infants were tested longitudinally, avoidance responses attenuated rather than increased (Campos, Hiatt, Ramsay, Henderson, \& Svejda, 1978; Eppler, Satterwhite, Wendt, \& Bruce, 1997). Thus, researchers are limited to between-subject designs with only a single trial per infant on each side. In addition to problems stemming from the safety glass, the fixed dimensions of the apparatus preclude conclusions about the accuracy of infants' responses and whether responses are scaled to each infant's current abilities.

\section{Summary: Surface Layout and Learning Sets}

Evidence from several developmental designs, various experimental arrangements with slopes and gaps, and psychophysical methods to normalize the relative risk to infants' bodies and skills supports a learning-set account of infants' ability to cope with novel and variable changes in the surface layout. The evidence is consistent with learning sets and inconsistent with association learning: Learning required weeks-or months-of everyday experience with the newly acquired postural-control system. Learning did not require encounters with particular cues in particular contexts; experienced infants could show adaptive responses with a novel obstacle on their first trial. Experienced infants showed broad transfer to novel variations in the surface layout and to changes in their own bodies and skills. And, infants showed no evidence of transfer between experienced postures and later developing ones.

\section{EVIDENCE FOR ASSOCIATION LEARNING: COPING WITH CHANGES IN FRICTION AND RIGIDITY}

Studies on infants' responses to variations in the friction and rigidity conditions of support surfaces present different methodological challenges than the research involving variations in the dimensions of the surface layout. With an adjustable apparatus, researchers can easily present infants with continuous changes in dimensions of the surface layout, such as gradations in the degree of slope or width of gap. In contrast, experimental manipulations of friction and rigidity conditions are difficult: They cannot be continuously adjusted by turning a crank or pulling a lever. Instead, material substance must be altered by presenting infants with different surfaces or different apparatuses. Moreover, even an infinitely large collection of surfaces would not guarantee continuous adjustments in friction or rigidity because the size of the emergent forces depends on infants' bodies and the manner of contact. Thus, researchers must rely on categorical rather than continuous comparisons of material substance: infants' responses to wobbly foam and rigid wooden handrails (Berger, Adolph, \& Lobo, 2005); deformable waterbed and rigid plywood surfaces (E. J. Gibson, Riccio, Schmuckler, Stoffregen, Rosenberg, \& Taormina, 1987); watery, rigid, elastic, and net surfaces (Ruff, 1984; Palmer, 1989; Bourgeois, Khawar, Neal, \& Lockman, 2005); and slippery, squishy, and sticky surfaces (Stoffregen, Adolph, Thelen, Gorday, \& Sheng, 1997). 
Here, we describe a new methodological approach designed to test prospective control of balance and locomotion in the context of association learning. We tested participants' motor decisions over a series of consecutive trials as they approached a single, well-marked, deformable or low-friction obstacle on a rigid, high-friction walkway. The novel changes in rigidity and friction conditions were created with large foam-filled blocks and a large Teflon insert respectively.

To encourage learning, the obstacles were marked with various types of visual cues that differentiated them from the surrounding walkway in color, texture, pattern, and shine. In the foam-pit paradigm, the foam blocks were bumpy with rounded edges and were covered with a brown and gold flowered, stretchy material. The rest of the walkway was covered with blue, smooth, and shiny vinyl. In the Teflon-floor paradigm, the Teflon insert was white, smooth, flat, squareedged, and shiny. The remainder of the walkway was covered with blue, plush, and textured carpet. Contrast trials (in which the foam pit was replaced with rigid wooden blocks and the Teflon was replaced with high-friction carpet) in a baseline condition served to highlight the visual cues that marked the challenging friction/ rigidity condition.

The obstacles ensured that participants would fall if they stepped haphazardly onto the foam or Teflon. In the foam-pit paradigm, the internal sections of the foam blocks were carved out so that the foam would suddenly depress several inches under the lightest infant's weight. In the'Teflon-floor paradigm, the entire walkway was tilted gently, and infants wore nylon stockings to ensure that they would slip if they did not change their ongoing walking patterns. An experimenter followed alongside participants to ensure their safety, and caregivers stood at the far side of the obstacle and encouraged them to cross.

The benefit of this methodological approach is that we could observe participants' exploratory activity and motor decisions on their first encounter with the obstacle to determine whether visual cues such as color, texture, pattern, and shine alert walkers to novel variations in rigidity or friction. In addition, if participants fell on the first trial, we could examine association learning over subsequent encounters with the obstacle over the test session. On an association-learning account, participants should pair the visual cues for the obstacle with the consequence of falling and avoid falling on subsequent encounters.

\section{Foam Pit}

In one series of experiments, we used a cross-sectional design to test age-related changes in association learning (Joh \& Adolph, 2006). We tested five groups of children (15-, 21-, 27-, 33-, and 39-month-olds) and a comparison group of adults on the walkway containing a foam pit (Fig. 7-5A). All of the participants were experienced at keeping balance in an upright postural control system and could be expected to show adaptive motor decisions to novel variations in the 3 months of walking the youngest infants (the 15-month-olds) had, on average, 3 months of walking experience. Thus, if forewarned, participants should also show adaptive responses to variations in rigidity and friction. In addition to the salient visual cues that distinguished the foam pit from the surrounding walkway,
A.

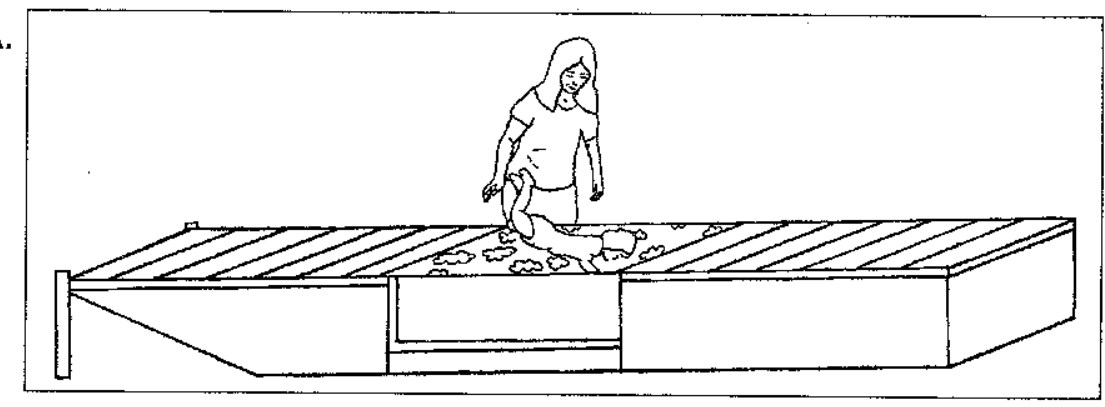

B.

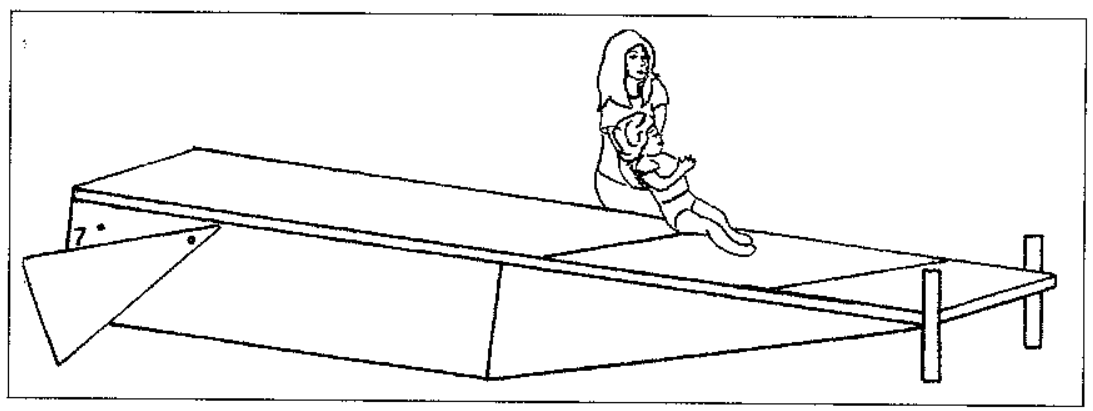

Figure 7-5. Tasks and apparatuses used in studies of association learning. (A) Crawling and walking infants, children, and adults falling on a walkway interrupted by a deformable foam pit. (B) Walking infants slipping on a walkway interrupted by a low-friction Teflon floor.

the consequences of falling were also highly salient. The foam pit was large enough that the younger children could fall headfirst into the foam without intervention by the experimenter. Experimenters caught the older children and assisted adults when they fell.

On their first trial, every participant walked straight into the foam pit and fell. In contrast to participants' performance in tasks involving novel variations in surface layout, everyday walking experience did not facilitate adaptive responses to novel variations in rigidity conditions. Visual cues for the foam pit-the bumpy surface and rounded edges of the foam blocks and the coincident change in the color, pattern, and texture of the material covering the foam pit-were not sufficient to elicit hesitation or focused exploration on the first encounter with the obstacle. In fact, across all ages, participants often gasped when they fell, indicating that the consequences were unexpected and salient.

The crucial test of learning, however, was the number of trials required to avoid falling on subsequent trials. The learning criterion was 2 consecutive no-fall trials. As shown in Figure 7-6, the 15-month-olds required 7 trials, on average, to meet the learning criterion. Only 2 out of 18 infants (11\%) showed 1 -trial learning (they avoided falling from their second trial) and 4 out of 18 infants (22\%) showed no evidence of learning (they fell on 16 consecutive foam-pit trials without pausing, 


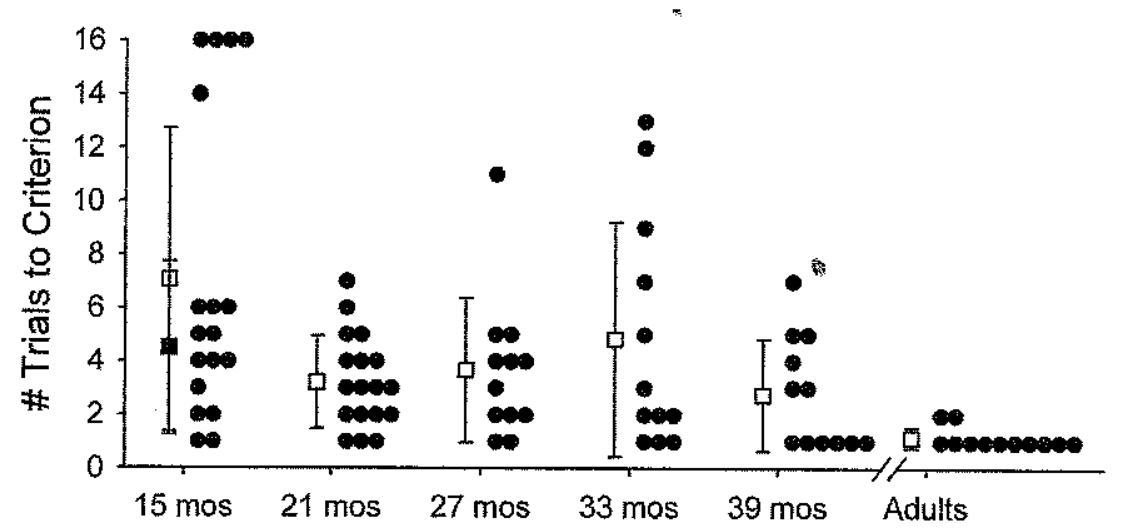

Figure 7-6. Number of trials required to meet the learning criterion ( 2 consecutive trials on which participants avoided falling) for walking infants, children, and adults in the foampit experiment. The circles represent individual data. The open squares represent group averages with all participants included. The filled square represents group average for the 15-month-old learners only.

exploring the foam pit, or testing alternative positions). With the non-learners excluded, the 15 -month-olds averaged 4.5 trials to meet the learning criterion.

Older children and adults fared better on two counts. No participants in the older age groups fell on every foam-pit trial. That is, all of the older children and adults met the learning criterion eventually during the testing session. In addition, the number of participants showing 1-trial learning increased with age, from $11 \%$ at 15 months to $50 \%$ at 39 months and $83 \%$ by adulthood.

However, two findings were similar across the age groups. First, learning was all-or-none: Before stepping onto the foam pit, participants rarely showed changes in hesitation, tactile exploration at the brink, or shifts in position until the learning criterion trials. For example, on their falling trials, 15 -month-olds required only 7 seconds to walk into the foam pit; and on the two no-fall learning criterion trials, infants spent 16 seconds on the platform before they embarked onto the foam pit using an alternative locomotor strategy. Within each age group, speed of learning was not predicted by duration of walking experience, the number of serious falls, gender, or weekly exposure to ground surfaces varying in material properties.

A second similarity across age groups is that learning to avoid falling did not involve a stereotyped motor response (Fig. 7-7). On trials in which they refused to walk into the foam pit, infants crossed in crawling, backing, and sitting positions, detoured off the side of the starting platform, appealed to the experimenter for help, or simply avoided traversal altogether. Older children sometimes employed a diving strategy in which they ran down the length of the walkway and dove into the foam pit. Adults leaped over the entire foam pit or stepped into it cautiously.

In addition, we used a different developmental design - a longitudinal studyto test transfer in association learning (Joh \& Adolph, 2005). Infants were observed on the foam pit apparatus every three weeks, from 10.5 months until 15 months
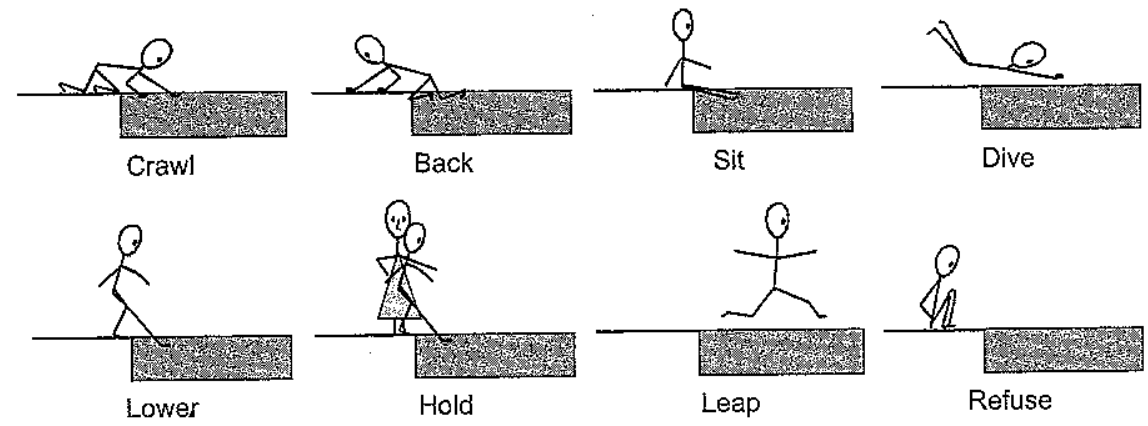

Figure 7-7. Various alternative locomotor strategies for avoiding falling into the foam pit.

of age. At the start of the study, all infants were crawlers; by the end of the study, all were walkers. This design allowed us to track the transfer of learning across 7 test sessions and across the transition from crawling to walking. To determine whether infants' responses were the result of experience with the foam pit, the 15-month-olds in the previous foam-pit experiment (Joh \& Adolph, 2006) served as an age-matched control. In addition, to test transfer of learning across contexts, we moved the foam pit to a different location in the lab. At infants' first walking session (infants were different ages) and the last testing session (all infants were 15 months old), after testing them on the foam pit in its original location, we moved the brown flowered covering to a different walkway.

Three sets of findings indicate that learning to avoid falling into the foam pit depended on context-specific associations between the visual cues for the foam pit and the consequence of falling rather than on the formation of a learning set. First, the number of trials to meet the learning criterion decreased steadily over the 7 test sessions. At their first session, at 10.5 months of age, infants averaged 10 consecutive falls before learning. At their last session, at 15 months of age, infants averaged only 4 falls. Across sessions, approximately a quarter of the infants never learned from falling. When the non-learners were excluded from the analysis, on their last session, infants required only 0.2 trials to learn from falling because 10 out of 19 infants avoided falling on the first trial, providing further evidence that they had remembered the visual cues for the foam pit from previous sessions. In comparison, the infants who were tested only once in previous work averaged 7 falls (4.5, excluding the non-learners) at 15 months of age.

The second important finding was that learning transferred over the developmental transition from crawling to walking postures. Because infants began walking at different points during the study, they were different ages and had different numbers of previous sessions at their first walking session. When the sessions were normalized to infants' first walking session, we found a steady decrease in the number of trials to learning criterion over weeks of crawling, over weeks of walking, and from infants' last crawling session to their first walking session. 
The third finding in support of association learning on the foam pit was that learning from falling was highly context-dependent. At infants' first walking session and last test session, we tested them on a different walkway in a different location in the laboratory, but otherwise retained the other aspects of the training context: the same visual cues for the foam-pit obstacle, the same experimenters, and the same toys and apparatuses in a cluttered laboratory. Moreover, the new walkway was less than 5 feet away from the location of the original walkway. Despite the minor change in the context, infants behaved as if they had not seen the foam pit previously on multiple occasions. Although the learners had avoided walking onto the foam pit a few minutes earlier, they stepped into it in the altered context without hesitation or tactile exploration.

\section{Slippery Teflon}

We replicated the foam-pit results with another group of 15 -month-olds on a slippery Teflon obstacle (Joh, Adolph, \& DeWind, 2005). In this case, the cues for the obstacle resembled ice (white, shiny, smooth, flat surface) but may have been less salient than the cues for the foam pit because the Teflon was not patterned, textured, or brightly colored (Fig. 7-5B). The consequences of falling were aversive but may have been less salient than those of falling face first into the foam pit. Because infants slipped backward as soon as they stepped onto the Teflon.insert, an experimenter caught them for safety as they began to fall.

As in the foam-pit experiments, visual cues to differentiate the novel surface from the surrounding walkway did not elicit focused exploration at the brink of the obstacle. And despite adults' reported reliance on shine to predict slip (Joh, Adolph, Campbell et al., 2006), every infant walked straight onto the Teffon surface and fell. Moreover, the lower salience of the Teflon experiment appeared to affect the speed of learning from falling. As a group, infants required 9.5 trials to learn to avoid falling (compared to 7 trials shown by the 15 -month-olds in the foam-pit experiment). However, when the non-learners were excluded, infants required 5 trials (equivalent to 4.5 trials in the foam-pit experiment), and the same percentage of infants learned after 1 trial $(11 \%)$, suggesting that salience did not affect the learners. Instead, salience seemed to affect the number of non-learners: Twice the number of infants failed to learn during the test session in the Teflon paradigm ( $44 \%$ ) compared to those in the foam-pit paradigm (22\%). As in the foam-pit experiment, hesitation, tactile exploration, and exploratory shifts in position increased only on the learning-criterion trials in the Teflon experiment, when infants had learned to link the visual cues for the Teflon with the consequence of falling.

\section{Summary: Friction and Rigidity and Association Learning}

The evidence from cross-sectional and longitudinal designs with the foam pit and slippery Teflon floor supports an association-learning account of prospective control of locomotion over changes in the rigidity and friction of the ground surface: Speed of learning was related to the salience of cues and consequences and the number of previous encounters with the particular cues and context. Learning was not related to the duration of everyday locomotor experience. Infants showed narrow transfer of learning to changes in the environmental context but no decrement in learning over the developmental transition from crawling to walking. Across age groups, learning was relatively fast and occurred within the course of a single test session. However, 1-trial learning increased with age, and the number of nonlearners decreased with age, indicating that infants take longer than older children and adults to associate the visible appearance of the ground with the consequence of falling.

\section{COPING WITH COVARIATIONS IN SURFACE LAYOUT AND FRICTION/RIGIDITY}

Frequently, changes in surface layout occur simultaneously with changes in friction and rigidity conditions. The slope in the road might be shallow or steep; the surface of the road might be dry or wet concrete. The snowdrift in the path might be large or small; the obstacle might be composed of tightly or loosely packed snow. In such cases, learning sets and association-learning mechanisms should operate in concert. The visual-depth cues for surface layout should elicit focused exploration of the obstacle and thereby generate perceptual information for rigidity and friction. Associated links between visual cues for material substance and consequences for balance should facilitate more adaptive responding with repeated encounters with the obstacle.

\section{Crossing Bridges with Wooden and Wobbly Handrails}

We used a bridge and handrail apparatus to examine infants' ${ }^{2}$ responses to covariations in surface layout and rigidity. Previous work showed that 16-month-olds can take two aspects of the surface layout into account when deciding whether to walk over a potential obstacle: the width of a bridge spanning a large crevice, and the presence of a solid handrail to augment balance on narrow bridges (Fig. 7-8A) (Berger \& Adolph, 2003). Infants' decisions to walk or not depended on both bridge width and handrail presence. On the wide bridges, infants walked straight across the bridge without hesitation, regardless of whether the handrail was available. However, on narrow bridges, motor decisions depended on handrail presence. When the handrail was absent, infants refused to leave the starting platform. When the handrail was available, infants used it to augment their balance. They turned sideways and walked slowly and carefully across the bridge, gripping the rail tightly with both arms.

Here, we introduced material substance as a factor by varying the rigidity of the handrail (Berger et al., 2005). The bridges were designed to pose a challenging problem in terms of surface layout: Narrow (10- and 20-cm) bridges were too difficult to walk across without a handrail to augment balance; on the wider $(40-\mathrm{cm})$ bridge, a handrail was unnecessary. The handrails were designed to provide a viable solution for crossing the narrow bridges, but only when the handrails were 

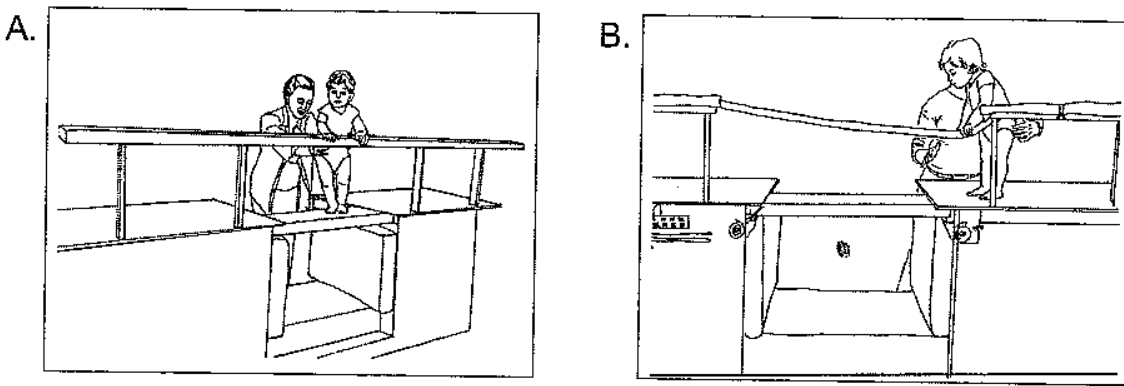

Figure 7-8. Task and apparatus used for studies of covariations in bridge width and handrail rigidity. (A) Infants walking across a bridge using a wooden handrail for support. (B) Infants walking across a bridge using wobbly rubber or foam handrails. The bridges varied in width $(12-72 \mathrm{~cm}$ for the wooden handrail task, $10-40 \mathrm{~cm}$ for the wobbly handrail task), changing infants' need to use handrails. All bridges were $74 \mathrm{~cm}$ long, and spanned a 76-cm deep crevice.

sufficiently rigid. On some trials, the handrail was made of sturdy wood, as in the earlier work, and it could support infants' weight. On other trials, the handrail was made of pliable rubber or foam and depressed to infants' knees when theydeaned on it (Fig. 7-8B). Visually, the three handrails were distinct: The wooden handrail was tan and had a wood-grain appearance, the rubber handrail was shiny yellow, and the foam handrail was matte blue. In addition, they were shaped differently: The wooden handrail had square edges whereas the wobbly ones had rounded edges. However, the rigidity of the handrail could be determined only by direct contact: The wobbly aspect of the rubber and foam rails could be revealed only by touch.

Infants took the rigidity of the handrail into account when deciding whether to walk. They crossed the narrow bridges more frequently on trials with the wooden handrail than on trials with the wobbly handrails. As predicted by learning sets, on trials with the narrow bridges, infants paused at the edge of the bridge and explored possible solutions for crossing the precipice by probing the bridge with their feet, exploring the handrail manually, and testing various ways of using the handrail to cross the bridge. They hesitated longer and spent more time engaged in tactile exploration (pushing, tapping, squeezing, rubbing, and even mouthing the handrail) on trials with the wobbly handrails. However, as predicted by association learning, infants showed evidence of learning over trials. On their second encounter with the wobbly handrails, infants showed less tactile exploration and their motor decisions were more discriminating, suggesting that they had linked the visual appearance of the handrail with the consequences for augmenting balance.

Two additional findings were unexpected. Although the wobbly handrails were designed to be functionally equivalent to having no handrail available for augmenting balance, infants attempted the narrow bridges at surprisingly high frequencies on trials with the wobbly handrails. A related surprise was that their attempts were largely successful. Infants managed to use the deformable rails

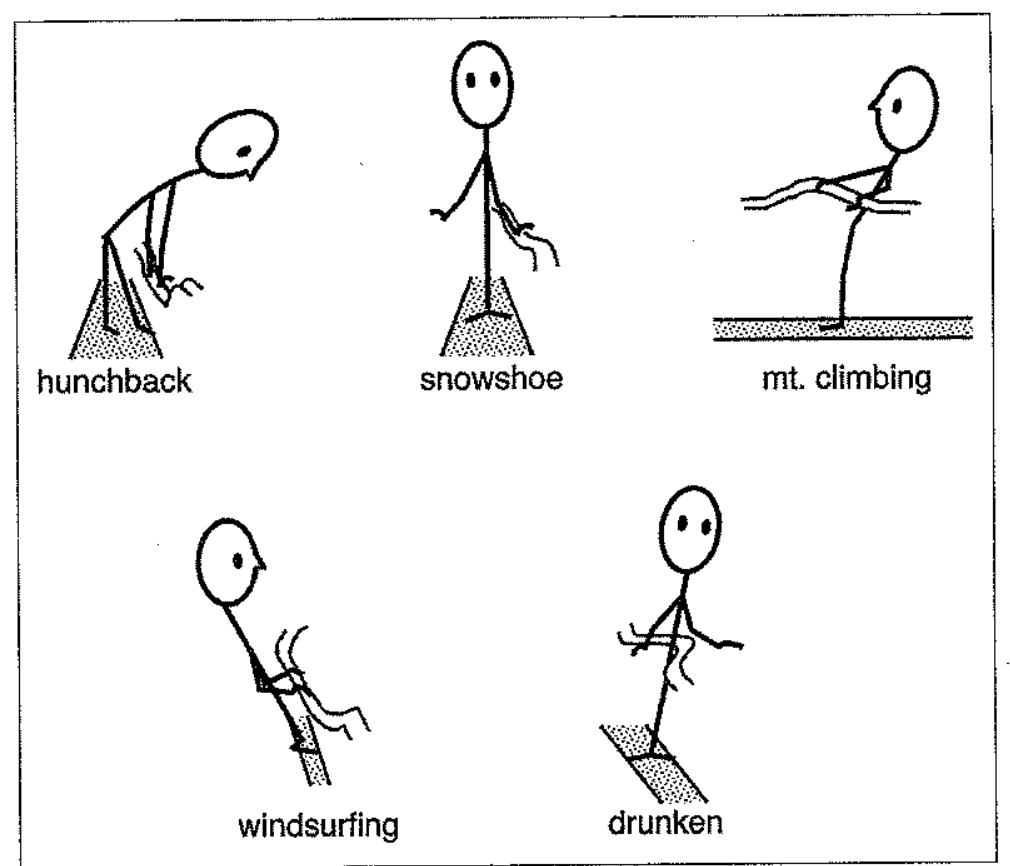

Figure 7-9. Strategies infants invented to use the wobbly rubber and foam handrails to help them walk over the narrower bridges.

adaptively by employing inventive strategies shown in Figure 7-9. For example, with a "hunchback" strategy, they walked sideways, stooped over, pressing down on the handrail so that it depressed to their knees; with a "mountain climbing" strategy, they faced forward, learned backward, and pulled up on the handrail like a rope, dragging themselves along hand over hand. Thus, even when association learning played a role in guiding infants' subsequent exploration and motor decisions, as in our previous work, their responses were flexible and varied, rather than rigid and stereotyped.

\section{Using Friction Underfoot to Guide Locomotion Over Slopes}

To examine infants' responses to covariations in surface layout and friction condition, we covered the adjustable sloping apparatus with high- and low-friction surfaces. The benefit of this method was that we could retain the precision of the psychophysical procedures used in earlier studies of surface layout. Specifically, we examined whether walkers extrapolate from incidental tactile information about friction that they feel beneath their feet on flat ground to visual information about upcoming changes in surface slant. Infants were tested on the same adjustable sloping walkway used in previous studies of learning sets (e.g., Adolph, 1997), and adults were tested on a sturdier version that had the same dimensions. 
In the high-friction condition, the entire walkway was covered with a novel rubber surface; in the low-friction condition, it was covered with a novel vinyl surface. Both surfaces were visually similar (shiny blue) to minimize the likelihood that participants could associate the appearance of the surface coverings with the consequences for maintaining balance. Participants wore nylon tights to exaggerate the effects of the rubber and vinyl and to keep footwear constant across participants. We used the psychophysical staircase procedure to estimate motor thresholds for each participant in each friction condition and presented additional probe trials on safe and risky increments to assess the adaptiveness of their motor decisions.

As in the lead-weighted shoulder pack study (Adolph \& Avolio, 2000), we examined the extent of transfer within an experienced postural-control system. In two experiments, we tested 14-month-old walking infants on the adjustable slope under high- and low-friction conditions (Lo, Avolio, Massop, \& Adolph, 1999; Adolph et al., 2008). In the furst experiment, high- and low-friction trials were interspersed, and in the second experiment, friction conditions were blocked. In both experiments, infants could feel their feet slip or grip the ground as they walked across the flat starting platform toward the slope. The critical questions were whether underfoot information about friction prompted focused visual and tactile exploration at the brink of the slope and whether infants' motor decisions were based on perceptual information about friction, slope, or both.

The results of both experiments were similar, regardless of whether friction conditions were interspersed or blocked. Infants' ability to walk down slopes was severely compromised in the low-friction condition: They could manage only 0 to 6-degree slopes without slipping and falling. Every infant could walk down steeper slopes in the high-friction condition: Motor thresholds ranged from 6 to 26 degrees. Most important, infants' motor decisions were based primarily on the degree of slope, not on friction. Despite feeling their feet slip on the flat starting platform, infants showed depressed levels of exploration in the low-friction condition. Without sufficient perceptual information, infants walked over the brink of risky low-friction slopes and fell.

In contrast to their dismal performance in the low-friction condition, infants' behavior in the high-friction condition replicated findings from previous studies with 14-month-olds on slopes (Adolph et al., 1993; Adolph, 1995, 1997; Adolph \& Avolio, 2000). Infants paused to explore slopes steeper than their motor thresholds by looking and touching before initiating descent. Consequently, they generated sufficient information to support an adaptive avoidance response. Normalized data showed that infants attempted risky low-friction slopes far beyond their abilities, but they scaled their attempts on high-friction trials to the conditional probability of success. When analyzed by absolute degree of slope, high- and low-friction curves were superimposed, meaning that infants' motor decisions were based on surface slant, not friction.

Several follow-up experiments showed that adults, like infants, do not incorporate underfoot information about friction into their decisions for walking down upcoming slopes (Joh, Adolph, Narayanan et al., 2007). Adults stood on a flat starting platform feeling the low-friction vinyl or high-friction rubber beneath their feet and looked at an adjustable slope a few steps ahead. They grossly overestimated their ability to cope with the low-friction surface and underestimated their abilities on the high-friction surface. But, when adults were instructed to generate the appropriate tactile information by placing part of their foot over the brink-so that they could obtain information about slant and friction simultaneously - their motor decisions matched their abilities.

To summarize, without distinct visual cues to differentiate friction conditions, walkers cannot form associations between the appearance of the ground surface and the consequences for locomotion. Both infant and adult walkers were aware that the slippery vinyl surface was more difficult; as in earlier work, their reactive adjustments were appropriately geared to the friction conditions (Stoffregen et al., 1997). In low-friction trials, they walked more slowly and stiffly upright toward the brink of the slope. However, neither infants nor adults appeared to realize that the feeling of slip on flat ground presaged dire consequences on a small, upcoming slope. In both high- and low-friction trials, when infants stopped at the brink to engage in tactile exploration, their motor decisions reflected the actual possibilities for walking successfully. The problem was that only the visual cues for surface slant prompted walkers to engage in tactile exploration at the brink. If the surface of the slope had been visually distinct from the rest of the walkway (e.g., a different color), as the wobbly handrails had been, infants may have eventually associated the visual cues with slipping and increased the frequency of exploratory touching on shallow slopes in the low-friction condition.

\section{CONCLUSIONS}

When people speak of an infant "learning" to walk, what they probably have in mind is an infant taking wobbly steps across the room. However, learning to walk is no more about producing stepping movements than learning to talk is about producing vocal sounds. Walking-like talking-involves producing the appropriate behaviors to fit the requirements of the current situation. Thus, infants have truly learned to walk when they can steer through a cluttered room, accommodate their movements to the requirements of different surface properties, modify their actions in accordance with the continual variations in their own bodies and skill levels, and select the appropriate responses from their repertoire. To be functionally useful, walking must be adapted to the constraints of the environment and the body. In short, learning to walk means perceiving and using affordances for locomotion adaptively.

\section{Exploration for Action}

Sitting, crawling, cruising, and walking are not just motor skills-they are perceptual-motor skills. Infants need relevant perceptual information to guide their motor actions adaptively. Exploratory movements of the eyes, head, and body generate the necessary perceptual information. Visual information from a distance is especially important because gross motor skills involve large displacements 
of the body and errors can have serious consequences for maintaining balance. Visual depth cues are a reliable source of information about novel variations in the surface layout. With visual depth cues, infants have sufficient time to explore upcoming obstacles and to plan a reasonable course of action. In contrast, novel variations in friction and rigidity are not reliably specified by visual cues from a distance because they are emergent forces. The relevant peyceptual information is created only during an event involving both contact surfaces (e.g., rubbing the foot against the brink of a slope). Without contact between the foot and the obstacle, only associations formed during prior encounters can link arbitrary visual cues with the consequences for balance and locomotion.

We suggest that differences in the availability of visual information from a distance create a reliance on different learning mechanisms: learning sets for surface layout and association learning for friction and rigidity. In principle, learning sets alone could be sufficient for coping with both surface layout and friction and rigidity if infants were willing to employ all of the exploratory procedures in their repertoires in response to arbitrary visual cues such as color, texture, and shine. However, in practice, infants and adults dispense their exploratory behaviors more efficiently. They continue on a current course of action unless visual depth cues alert them to a potential obstacle, or prior encounters with a similar surface in a similar situation were linked with a loss of balance. Thus, infants and adults must rely on association learning as a fallback mechanism for guiding prospective control under variable conditions of friction and rigidity. The cost of economy, however, is that they also must incur a high frequency of falls on their first encounter with a slippery or squishy surface.

\section{Learning in Development}

We also suggest that studies of infants maintaining balance in stance and locomotion under challenging and novel conditions provide a useful model system for investigating general principles of learning and development. In terms of learning, our studies of infants walking down slopes, leaning over gaps, and so on indicate that psychological functions with a high demand for adaptability and flexibility are best served by a learning mechanism that facilitates broad transfer of learning. Adaptability and flexibility are critical for maintaining balance in stance and locomotion because the constraints on balance are continually changing. Learning sets, albeit slow and difficult to acquire, are an optimal learning mechanism for ensuring broad transfer.

In terms of development, these studies provide a dramatic example of psychological functions that are ontogenetic adaptations, that is, functions specific to particular periods of development. For years, research from developmental systems theory has stressed the view that infants are not miniature adults (Oppenheim, 1981, 1984). Many of infants' behaviors are adaptations to their particular developmental niche. The studies described in this chapter suggest that crawlers are not little walkers. Earlier appearing postural-control systems involve qualitatively different psychological functions from later developing ones. Learning to maintain balance in a sitting posture does not teach infants to maintain balance while crawling; crawling does not ensure adaptive responding while walking; and even cruising, which shares the similarity of upright posture with walking, does not teach infants to respond adaptively to novel changes in the ground surface. Sitting, crawling, cruising, and walking show four separate learning curves after the onset of each postural milestone.

Finally, the studies described in this chapter provide insights into the ontogeny of learning. That is, learning occurs in development. Infants acquire learning sets and paired associations in the larger context of ongoing developmental changes. The two processes are linked in the sense that learning and development are concurrent, but developmental changes need not be specific to the type of learning mechanism. employed or to the nature of what is being learned. Developments in one domain can serendipitously constrain and/or facilitate learning in a different domain.

We have suggested that the ongoing developmental changes in infants' bodies, skills, and environments may facilitate the acquisition of learning sets by providing infants with immense amounts of practice with continually varying and novel stimuli. Reciprocally, infants' episodic physical growth, fluctuating motor skills, and shifting landscapes may constrain them from forming associations between particular facts about their bodies or environments and the consequences for postural control. Yesterday's body size may be different from the size of the body today. Poor crawling skill last week may be replaced by proficient crawling this week. The high frequency of infants' falls and near falls may lead them to ignore the particular appearance of the ground suxface. Our studies of balance and locomotion in infants suggest that the nested structure of learning in development may be responsible for selecting the optimal learning mechanisms for each psychological function.

Acknowledgments We thank Mark Blumberg for his helpful suggestions and comments. We also thank Lana Karasik, Jessie Garciaguirre, and Michael Smith for their assistance with the figures. Work on this chapter was supported by National Institute of Health grants HD-33486 and HD-42697 to Karen Adolph.

References

Adolph, K. E. (1995). A psychophysical assessment of toddlers' ability to cope with slopes. Journal of Experimental Psychology: Human Perception and Performance, 21, $734-750$.

Adolph, K. E. (1997). Learning in the development of infant locomotion. Monographs of the Society for Research in Child Development, 62(3, Serial No. 251).

Adolph, K. E. (2000). Specificity of learning: Why infants fall over a veritable cliff. Psy. chological Science, 11, 290-295.

Adolph, K. E. (2002). Learning to keep balance. In R. Kail (Ed.), Advances in child development and behavior (Vol. 30, pp. 1-30). Amsterdam: Elsevier Science.

Adolph, K. E. (2005). Learning to learn in the development of action. In J. Lockman \& J. Reiser (Eds.), Action as an organizer of learning and development: The 32nd Minnesota Symposium on Child Development (pp. 91-122). Hillsdale, NJ: Lawrence Erlbaum. 
Adolph, K. E., \& Avolio, A. M. (2000). Walking infants adapt locomotion to changing body dimensions. Journal of Experimental Psychology: Human Perception and Performance, 26, 1148-1166.

Adolph, K. E., \& Berger, S. E. (2005). Physical and motor development. In M. H. Bornstein \& M. E. Lamb (Eds.), Developmental science: An advanced textbook (5th ed. pp. 223-281). Mahwah, NJ: Lawrence Erlbaum.

Adolph, K. E., \& Berger, S. E. (2006). Motor development. In DaKuhn \& R. S. Siegler (Eds.), Handbook of child psychology: Vol. 2: Cognition, perception, and language (6th ed., pp. 161-213). New York: John Wiley.

Adolph, K. E., \& Eppler, M. A. (2002). Flexibility and specificity in infant motor skill acquisition. In J. W. Fagen \& H. Hayne (Eds.), Progress in infancy research (Vol. 2 , pp. 121-167). Mahwah, NJ: Lawrence Erlbaum.

Adolph, K. E., Eppler, M. A., \& Gibson, E. J. (1993). Crawling versus walking infants' perception of affordances for locomotion over sloping surfaces. Child Development, $64,1158-1174$

Adolph, K. E., Eppler, M. A., Marin, L., Weise, I. B., \& Clearfeld, M. W. (2000). Exploration in the service of prospective control. Infant Behavior and Development, 23, $441-460$

Adolph, K. E., Joh, A. S., \& Eppler, M. A. (2008). Infants' perception of affordances of slopes under high and low friction conditions. Manuscript submitted for publication.

Adolph, K. E., Tamis-LeMonda, C. S., Ishak, S., Karasik, L. B., \& Lobo, S. A. (2008). Locomotor experience and use of social information are posture specific. Manuscript submitted for publication.

Baillargeon, R. (2004). Infants' reasoning about hidden objects: Evidence for eventgeneral and event-specific expectations. Developmental Science, 7, 391-424.

Baker, A. G., \& Mackintosh, N. J. (1979). Preexposure to the CS alone, US alone, or CS and US uncorrelated: Latent inhibition, blocking by context or learned irrelevance? Learning and Motivation, 10, 278-294.

Berger, S. E., \& Adolph, K. E. (2003). Infants use handrails as tools in a locomotor task. Developmental Psychology, 39, 594-605.

Berger, S. E., Adolph, K. E., \& Lobo, S. A. (2005). Out of the toolbox: Toddlers differentiate wobbly and wooden handrails. Child Development, 76, 1294-1307.

Bertenthal, B. I., Campos, J. J., \& Barrett, K. C. (1984). Self-produced locomotion: Au organizer of emotional, cognitive, and social development in infancy. In R. N. Emde \& R. J. Harmon (Eds.), Continuities and discontinuities in development (pp. 175-210). New York: Plenum Press.

Bertenthal, B. I., \& Clifton, R. K. (1998). Perception and action. In D. Kuhn \& R. S. Siegler (Eds.), Handbook of child psychology: Vol. 2: Cognition, perception, and language (5th ed., pp. 51-102). New York: John Wiley.

Bourgeois, K. S., Khawar, A. W., Neal, S. A., \& Lockman, J. J. (2005). Infant manual exploration of objects, surfaces, and their interrelations. Infancy, 8, 233-252.

Bouton, M. E., \& Bolles, R. C. (1979). Contextual control of the extinction of conditioned fear. Learning and Motivation, 10, 445-466.

Campos, J. J., Hiatt, S., Ramsay, D., Henderson, C., \& Svejda, M. (1978). The emergence of fear on the visual cliff. In M. Lewis \& L. Rosenblum (Eds.), The development of affect (pp. 149-182). New York: Plenum.

Chan, M. Y., Biancaniello, R., Adolph, K. E., \& Marin, L. (2000, July). Tracking infants' locomotor experience: The telephone diary. Poster presented to the International Conference on Infant Studies, Brighton, UK.
Eppler, M. A., Satterwhite, T., Wendt, J., \& Bruce, K. (1997). Infants' responses to a visual cliff and other ground surfaces. In M. A. Schmuckler \& J. M. Kennedy (Eds.), Studies in perception and action $I V$ (pp. 219-222). Mahway, NJ: Lawrence Erlbaum.

Frankenburg, W. K., \& Dodds, J. B. (1967). The Denver Developmental Screening Test. Journal of Pediatrics, 71, 181-191.

Garciaguirre, J. S., \& Adolph, K. E. (2006, June). Infants'everyday locomotor experience: $A$ walking and falling marathon. Paper presented to the International Society for Infant Studies, Kyoto, Japan.

Gibson, E. J. (1988). Exploratory behavior in the development of perceiving, acting and the acquiring of knowledge. Annual Review of Psychology, 39, 1-41.

Gibson, E. J. (1992, April). Perceptual learning and development. Presentation to the Indiana University Psychology Department Colloquium, Bloomington, IN.

Gibson, E. J., \& Pick, A. D. (2000). An ecological approach to perceptual learning and development. New York: Oxford University Press.

Gibson, E. J., Riccio, G., Schmuckler, M. A., Stoffregen, T. A., Rosenberg, D., \& Taormina, J. (1987). Detection of the traversability of surfaces by crawling and walking infants. Journal of Experimental Psychology: Human Perception and Performance, 13, 533-544.

Gibson, E. J., \& Walk, R. D. (1960). The "visual cliff." Scientific American, 202, 64-71.

Gibson, J. J. (1979). The ecological approach to visual perception. Boston: HoughtonMifflin.

Haehl, V., Vardaxis, V., \& Ulrich, B. D. (2000). Learning to cruise: Bernstein's theory applied to skill acquisition during infancy. Human Movement Science, 19, 685-715.

Harlow, H. F. (1949). The formation of learning sets. Psychological Review, 56, 51-65.

Harlow, H. F. (1959). Learning set and error factor theory. In S. Koch (Ed.), Psychology: A study of a science (pp. 492-533). New York: McGraw-Hill.

Harlow, H. F., \& Kuenne, M. (1949). Learning to think. Scientific American, 3-6.

Hopkins, B., \& Westra, T. (1988). Maternal handling and motor development: An intracultural study. Genetic, Social and General Psychology Monographs, 114, 379-408.

Hopkins, B., \& Westra, T. (1989). Maternal expectations of their infants' development: Some cultural differences. Developmental Medicine and Child Neurology, 31, 384-390.

Hopkins, B., \& Westra, T. (1990). Motor development, maternal expectations, and the role of handling. Infant Behavior and Development, 13, 117-122.

Joh, A. S., \& Adolph, K. E. (2005, April). Crawling, walking, and learning about falling. Poster presented at the meeting of the Society for Research in Child Development, Atlanta, GA

Joh, A. S., \& Adolph, K. E. (2006). Learning from falling. Child Development, 77, 89-102.

Joh, A. S., Adolph, K. E., Campbell, M. R., \& Eppler, M. A. (2006). Why walkers slip: Shine is not a reliable cue for slippery ground. Perception \& Psychophysics, 68, 339--352.

Joh, A. S., Adolph, K. E., \& DeWind, N. K. (2005, November). Learning from slipping and falling. Poster presented at the meeting of the International Society for Developmental Psychobiology, Washington, DC.

Joh, A. S., Adolph, K. E., Narayanan, P. J., \& Dietz, V. A. (2007). Gauging possibilities for action based on friction underfoot. Journal of Experimental Psychology: Human Perception and Performance, 33, 1145-1157.

Johnson, S. P., Slemmer, J. A., \& Amso, D. (2004). Where infants look determines how they see: Eye movements and object perception performance in 3-month-olds. Infancy, 6 , 185-201.

Lampl, M., Veldhuis, J. D., \& Johnson, M. L. (1992). Saltation and stasis: A model of human growth. Science, 258, 801-803. 
Lashley, K. S. (1930). The mechanism of vision. I. A method for rapid analysis of patternvision in the rat. Journal of Genetic Psychology, 37, 453-360.

Lee, D. N. (1993). Body-environment coupling. In U. Neisser (Ed.), The perceived self: Ecological and interpersonal sources of self-knowledge (pp. 43-67). Cambridge: Cambridge University Press.

Leo, A. J., Chiu, J., \& Adolph, K. E. (2000, July). Temporal and functional relationships of crawling, cruising, and walking. Poster presented at the International Conference on Infant Studies, Brighton, UK.

Lewis, M., Alessandri, S. M., \& Sullivan, M. W. (1990). Violation of expectancy, loss of control, and anger expressions in young infants. Developmental Psychology, 26, $745-751$.

Lo, T., Avolio, A. M., Massop, S. A., \& Adolph, K. E. (1999). Why toddlers don't perceive risky ground based on surface friction. In M. A. Grealy \& J. A. Thompson (Eds.) Studies in perception and action V (pp. 231-235). Mahwah, NJ: Lawrence Erlbaum.

Marcus, G. F., Vijayan, S., Bandi Rao, S., \& Vishton, P. M. (1999). Rule learning by sevenmonth-olds infants. Science, 283, 77-80.

McGraw, M. B. (1935). Growth: A study of Johnny and Jimmy. New York: Appleton-Century.

Metcalfe, J. S., \& Clark, J. E. (2000). Sensory information affords exploration of posture in newly walking infants and toddlers. Infant Behavior and Development, 23, 391-405.

Mondschein, E. R., Adolph, K. E., \& Tamis-LeMonda, C. S. (2000). Gender bias in mothers' expectations about infant crawling. Journal of Experimental Child Psychology, 77, 304-316.

National Safety Council. (2003). Injury facts (B. Itasca, IL: National Safety Council.

Oppenheim, R. W. (1981). Ontogenetic adaptations and retrogressive processes in the development of the nervous system and behavior: A neuroembryological perspective. In K. J. Connolly \& H.F.R. Prechtl (Eds.), Maturation and development: Biological and psychological perspectives (pp. 73-109). Philadelphia: J. P. Lippincott.

Oppenheim, R. W. (1984). Ontogenetic adaptations in neural and behavioral development: Toward a more "ecological" developmental psychology. In H.F.R. Prechtl (Ed.), Continuity of neural functions from prenatal to postnatal life (pp. 16-30). Oxford: Blackwell Scientific Publications.

Palmer, C. F. (1989). The discriminating nature of infants' exploratory actions. Developmental Psychology, 25, 885-893.

Patla, A. E. (1989). In search of laws for the visual control of locomotion. Journal of Experimental Psychology: Human Perception and Performance, 15, 624-628. Patla, A. E. (1998). How is human gait controlled by vision? Ecological Psychology,
10, 287-302.

Patla, A. E., Prentice, S. D., Robinson, C., \& Neufeld, J. (1991). Visual control of locomotion: Strategies for changing direction and for going over obstacles. Journal of Experimental Psychology, 17, 603-634.

Patla, A. E., Robinson, C., Samways, M., \& Armstrong, C. J. (1989). Visual control of step length during overground locomotion: Task-specific modulation of the locomotor synergy. Journal of Experimental Psychology, 15, 603-617.

Rader, N., Bausano, M., \& Richards, J. E. (1980). On the nature of the visual-cliff-avoidance response in human infants. Child Development, 51, 61-68.

Reed, E. S. (1982). An outline of a theory of action systems. Journal of Motor Behavior, 14 98-134.

Reed, E. S. (1989). Changing theories of postural development. In M. H. Woollacott \& A. Shumway-Cook (Eds.), Development of posture and gait across the lifespan (pp. 3-24). Columbia, SC: University of South Carolina Press.
Richards, J. E., \& Rader, N. (1981). Crawling-onset age predicts visual cliff avoidance in infants. Journal of Experimental Psychology: Human Perception and Performance, 7, 382-387.

Richards, J. E., \& Rader, N. (1983). Affective, behavioral, and avoidance responses on the visual cliff: Effects of crawling onset age, crawling experience, and testing age. $P s y-$ chophysiology, 20, 633-642.

Rovee, C. K., \& Rovee, D. T. (1969). Conjugate reinforcement of infant exploratory behavior. Journal of Experimental Child Psychology, 8, 33-39.

Ruff, H. A. (1984). Infants' manipulative exploration of objects: Effects of age and object characteristics. Developmental Psychology, 20, 9-20.

Saffran, J. R. (2003). Statistical language learning: Mechanisms and constraints. Current Directions in Psychological Science, 12, 110-114.

Saffran, J. R., Aslin, R. N., \& Newport, E. L. (1996). Statistical learning by 8-month-olds infants. Science, 274, 1926-1928.

Siegler, R. S., Adolph, K. E., \& Lemaire, P. (1996). Strategy choice across the life span. In L. M. Reder (Ed.), Implicit memory and metacognition (pp. 79-121). Mahwah, NJ: Erlbaum.

Smith, L. B., \& Yoshida, H. (2005). Linguistic cues enhance the learning of perceptual cues. Psychological Science, 16, 90-95.

Sorce, J. F., Emde, R. N., Campos, J. J., \& Klinnert, M. D. (1985). Maternal emotional signaling: Its effects on the visual cliff behavior of 1-year-olds. Developmental Psychology, 21, 195-200.

Stevenson, H. W. (1972). Children's learning. New York: Appleton-Century-Crofts.

Stoffregen, T. A., Adolph, K. E., Thelen, E., Gorday, K. M., \& Sheng, Y. Y. (1997). Toddlers' postural adaptations to different support surfaces. Motor Control, 1, 119-137.

von Hofsten, C. (2003). On the development of perception and action. In K. J. Connolly \& J. Valsiner (Eds.), Handbook of developmental psychology (pp. 114-140), London: Sage.

von Hofsten, C. (2004). An action perspective on motor development. Trends in Cognitive Sciences, 8, 266-272.

Wang, S., Baillargeon, R., \& Brueckner, L. (2004). Young infants' reasoning about hidden objects: Evidence from violation-of-expectation tasks with test trials. Cognition, 93, 167-198.

Witherington, D. C., Campos, J. J., Anderson, D. I., Lejeune, L., \& Seah, E. (2005). Avoidance of heights on the visual cliff in newly walking infants. Infancy, 7, 285-298. 